\title{
Insecticidal and Enzyme Inhibition Activities of Leaf/bark Extracts, Fractions, Seed Oil and Isolated Compounds From Triadica Sebifera (L.) Small Against Aphis Craccivora Koch
}

Shudh Kirti Dolma

Institute of Himalayan Bioresource Technology

Prithvi Pal Singh

Institute of Himalayan Bioresource Technology

S. G. Eswara Reddy ( $\sim$ ereddy2001@yahoo.com )

Institute of Himalayan Bioresource Technology

\section{Research Article}

Keywords: Chinese tallow, insecticidal activities, synergistic, detoxification, AChE, GST

Posted Date: November 23rd, 2021

DOI: https://doi.org/10.21203/rs.3.rs-1060957/v1

License: (9) This work is licensed under a Creative Commons Attribution 4.0 International License.

Read Full License

Version of Record: A version of this preprint was published at Molecules on March 18th, 2022. See the published version at https://doi.org/10.3390/molecules27061967. 


\section{Abstract}

Aphid, Aphis craccivora Koch (Hemiptera: Aphididae) is a major sap-sucking insect pest of leguminous crops and also transmit plant viruses leading to economic yield loss. Insecticidal activities of $T$. sebifera have not been reported against $A$. craccivora and other insect pests till date. In this study, residual toxicity of leaf/bark extracts, seed oil, fractions, isolated compounds and its combinations; synergistic activity of leaf/ bark extract with seed oil and its enzyme inhibition activities against $A$. craccivora was studied. Among extracts, ethanolic bark extract $80 \%\left(\mathrm{LC}_{50}=5115.98 \mathrm{mg} \mathrm{L}^{-1}\right)$ was more effective against $A$. craccivora than other extracts. Among fractions, $n$-hexane fraction of leaf $\left(\mathrm{LC}_{50}=425.73 \mathrm{mg} \mathrm{L}^{-1}\right)$ and ethyl acetate fraction of bark $\left(\mathrm{LC}_{50}=813.45 \mathrm{mg} \mathrm{L}^{-1}\right)$ was more effective. Among compounds, gallic acid was most effective $\left(\mathrm{LC}_{50}=1303.68 \mathrm{mg} \mathrm{L}^{-1}\right)$ than shikimic acid and quercetin $\left(\mathrm{LC}_{50}=1725.09\right.$ and $1855.93 \mathrm{mg} \mathrm{L}^{-}$ ${ }_{1}^{1}$, respectively). Seed oil $\left(\mathrm{LC}_{50}=850.94 \mathrm{mg} \mathrm{L}^{-1}\right)$ is more superior than extracts/fractions/compounds. All the combinations showed toxicity and synergistic activity. Leaf/bark extracts and seed oil (0.25-2 $\left.\mathrm{mg} \mathrm{L}^{-1}\right)$ significantly inhibits the AChE and GST activity in A. craccivora. Based on field bio-efficacy studies, the leaf extract/seed oil or their combinations can be recommended for development of botanical formulation for the control of aphid.

\section{Introduction}

Aphid, Aphis craccivora Koch (Hemiptera: Aphididae) is the major sap-sucking insect pest of leguminous crops. Adults and nymphs suck the plant sap from leaves/stem/pods which affects the growth. In severe infestation, stunted growth of plants, secretion of honeydew on leaves/pods which attracts the development of sooty mould fungus which affects the photosynthesis of plants. A. craccivora also transmits plant viruses leading to economic yield loss ${ }^{1}$. Currently, farmers are using synthetic insecticides of different classes for the control of aphids. Non-judicious and repeated application of insecticides for control of aphids led to the development of resistance ${ }^{2}$. Due to the harmful effect of chemical insecticides onthe environment, public, customers, non-target organisms, there is an essential substitute for the effective management of aphid.

Chinese tallow, Triadica sebifera (L.) Small (Euphorbiaceae) is the world's most invasive species common in China ${ }^{3}$. It is used for traditional medicine in China, Taiwan, and Japan. Due to the rapid growth, widespread invasion of native ecosystems, still it is used as a popular ornamental due to its beautiful foliage that changes from yellow to crimson colour. Seeds were used for herbal medicines, candles, soap, cocoa butter, lamps, heating oils, cosmetics, and pharmaceuticals ${ }^{4-5}$. The seed oil is used to treat skin disorders, purgatory, and root bark decoctions caused by dyspepsia ${ }^{6}$.The leaves and roots having the properties of depurative, diuretic, and laxative. The decoction is used to cure edema, constipation. The root and bark extracts are used for the treatment of snake bites and skin ulcers ${ }^{7}$. T. sebifera has the properties of anti-bacterial, anti-viral, antioxidant, analgesic, anti-inflammatory, anti-hypersensitivity ${ }^{8-11}$. 
Insecticidal activities of $T$. sebifera have not been reported against aphid and other insect pests till date. Therefore, in the present study, we have explored T. sebifera for 1 ). Chemical analysis of $n$-hexane fractions of leaf/bark ethanolic aqueous extracts of T. sebifera; 2). Isolation of compounds from ethyl acetate and $n$-butanol fractions of leaf; 3 ). Residual toxicity of leaf/bark ethanolic/methanolic aqueous extracts, seed oil and its combinations, fractions, isolated compounds; 4). Combination of seed oil with leaf/bark extracts for its synergistic interaction against $A$. craccivora and 5). Detoxification enzyme activities of LEE, BEE and SO against $A$. craccivora

\section{Methods}

\section{Plant material}

The leaves at the vegetative stage, bark, and seeds of $T$. sebifera used in the study was collected in and around CSIR-IHBT Campus, Palampur, Himachal Pradesh (32 $\left.06^{\prime} 05^{\prime \prime} \mathrm{N}, 76^{\circ} 34^{\prime} 10^{\prime \prime} \mathrm{E}\right)$ located in the Dhauladhar range of the western Himalayas. The authentication of plant material was done by a Taxonomist of CSIR-IHBT, Palampur (H.P.), India, and voucher specimen were deposited in the herbarium (voucher no. PLP 18563) of the institute. The plant material was dried under shade for 15 days and was used for further processing.

\section{Statement to confirm the methods:}

All methods were carried out in accordance with relevant guidelines and regulations.

\section{Statement for collection of the plant material}

Institutional permission was taken for the collection of plant material and the voucher specimens (PLP 18563) were deposited in the herbarium of the institute.

\section{Preparation of leaf/bark extracts and fractions}

The well-dried leaves and bark samples were powdered using a grinder. About $1 \mathrm{~kg}$ of samples was extracted separately by maceration ( $3 \times 5 \mathrm{~L}$ ) $12 \mathrm{~h}$ intervals using a concentration of $50 \%, 80 \%$ and $100 \%$ ethanol: water at room temperature. The macerated samples were filtered, and then the solvent was removed under low pressure at 45 $₫$ Cin a Rotatory evaporator (Buchi, R-210), accordingly, leaf/bark, ethanolic/methanolic extracts at a concentration of $50 \%, 80 \%$, and $100 \%$ were also prepared. The yields obtained for $50 \%, 80 \%$ and $100 \%$ leaf ethanolic aqueous extracts (LEE) were $78.8,90.5$, and $51.4 \mathrm{~g}$, respectively. Similarly, the yield obtained for $50 \%, 80 \%$ and $100 \%$ leaf methanolic aqueous extracts (LME) were $77.3 \mathrm{~g}, 72.6 \mathrm{~g}$, and $48.6 \mathrm{~g}$, respectively. The yield obtained for $50 \%, 80 \%$ and $100 \%$ bark ethanolic aqueous extracts (BEE) were $77.5,85.6$, and $53.2 \mathrm{~g}$, respectively. In the same way, the yield obtained for $50 \%, 80 \%$ and $100 \%$ bark methanolic aqueous extracts (BME) were $67.5 \mathrm{~g}, 78.5 \mathrm{~g}$, and $63.3 \mathrm{~g}$, respectively, and kept $<4 \llbracket C$ until further analysis starts. 
The dried ethanolic aqueous extract $80 \%$ from leaf $(75 \mathrm{~g})$ and bark $(65 \mathrm{~g})$ was dissolved in a minimum volume of distilled water and sequentially fractionated $(500 \mathrm{~mL} \times 5)$ from lower to higher polarity of the

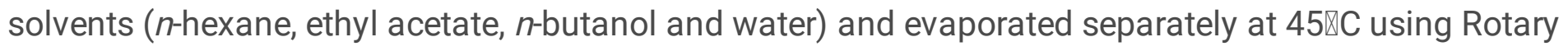
evaporator and then lyophilized to get respective fractions. The yields obtained for the $n$-hexane, ethyl acetate, $n$-butanol and water fractions of leaf were $25 \mathrm{~g}, 21.5 \mathrm{~g}, 18.4 \mathrm{~g}$ and $8.1 \mathrm{~g}$, respectively. Similarly, the yields obtained for the $n$-hexane, ethyl acetate, $n$-butanol and water fractions of bark were $10 \mathrm{~g}, 8.58$ g, $19.7 \mathrm{~g}$ and $8.3 \mathrm{~g}$, respectively and kept $<4 \rrbracket \mathrm{C}$ until further analysis (Fig. 2).

\section{Extraction of seed oil}

About $10 \mathrm{~kg}$ seeds were dried under the shade for 15 days. The seed coat was stripped manually, defatted by mixing them in hot water $(70 \otimes \mathrm{C})$ using a mini extractor. The defatting process was regulated in the mini extraction system for $3.5 \mathrm{~h} /$ percolation 6 times, for the appearance of black kernels and then cleaned with hot water then transferred to a hot air oven $(45 \otimes C)$ for drying. When the kernels were dehydrated, $2 \mathrm{~kg}$ of kernels were grounded in a blender and dissolved in $n$-hexane ( $3 \mathrm{~L} \times 4$ times) in a percolator. The eluted solvent was collected, filtered, and removed under low pressure at 45区C in Rotavapor (Buchi, R-210) which yields $1 \mathrm{~L}$ of seed oil (SO) which was kept in $<4 \llbracket C$ until further analysis.

\section{Isolation of compounds}

The ethyl acetate fraction $(8 \mathrm{~g})$ was taken and subjected for column chromatography over 60-120 mesh size silica gel. The column was packed in pure chloroform and eluted with methanol in the increasing order of polarity. A total of 50 fractions were collected $(100 \mathrm{ml}$ each) using a gradient elution of chloroform and methanol mixture. All the fractions were then divided into seven main fractions (i.e., $\mathrm{F}_{\mathrm{A}^{-}}$ $\mathrm{F}_{\mathrm{G}}$ ) after TLC analyses. Fractions $\mathrm{F}_{\mathrm{C}}-\mathrm{F}_{\mathrm{D}}$ eluted at polarity $(20: 80) \mathrm{MeOH}: \mathrm{CHCl}_{3}$ and then again subjected for column chromatography over 60-120 mesh size silica gel, results in the isolation of compound 1 (128 $\mathrm{mg})$ at polarity $(10: 90) \mathrm{MeOH}: \mathrm{CHCl}_{3}$ and compound $2(150 \mathrm{mg})$ at polarity $(15: 85) \mathrm{MeOH}: \mathrm{CHCl}_{3}$.

$n$-butanol fraction $(6 \mathrm{~g})$ was subjected to column chromatography for isolation of pure molecules. Mesh size 60-120 silica was used with solvent system chloroform: methanol: formic acid for column chromatography. At different polarities and TLC-based analysis, five major fractions (i.e., $\mathrm{BF}_{\mathrm{A}}-\mathrm{BF}_{\mathrm{E}}$ ) were collected. $\mathrm{BF}_{\mathrm{A}}$ fraction was again subjected to 230-400 silica for isolation. The solvent system used for isolation was chloroform: methanol with $0.01 \%$ formic acid. Compound $\mathbf{3},(124 \mathrm{mg})$ was isolated at $3 \%$ methanol: chloroform with $0.01 \%$ formic acid and compound $\mathbf{4}(108 \mathrm{mg}$ ) was isolated at $5 \%$ methanol: chloroform with $0.01 \%$ formic acid.

\section{Preparation of fatty acid methyl esters (FAMES)}

The FAMES were prepared as per the method followed by Adebisi et al. ${ }^{22}$. Briefly, $1 \mathrm{~mL}$ of $n$-hexane was added into $0.1 \mathrm{~mL}$ oil and $1 \mathrm{~mL}$ of sodium methoxide $(1.5 \mathrm{~g} \mathrm{NaOH}$ in $50 \mathrm{~mL}$ methanol). The mixture was stirred vigorously using vortex and kept untouched for 10 min to segregate the transparent fatty acid methyl ester solution from the opaque aqueous layer and was stored at $4 \llbracket \mathrm{C}$ until further use. 
Gas chromatography-mass spectrophotometry (GC-MS) analysis

The GC-MS analysis of $n$-hexane fractions of leaf and bark ethanolic aqueous extracts and seed oil were carried out using a Shimadzu QP 2010 and DB-5MS (J \& W Scientific, Folsom, USA) capillary column (30 $\mathrm{m} \times 0.25 \mathrm{~mm}$, i.d., $0.25 \mu \mathrm{m}$ thickness). The $\mathrm{GC}$ oven temperature was programmed at $70 \otimes \mathrm{C}$ for $4 \mathrm{~min}$ and then increased to $220 \varangle \mathrm{C}$ at the $4^{\circ} \mathrm{C} / \mathrm{min}$ and held for $5 \mathrm{~min}$. Injector temperature was $240 \otimes \mathrm{C}$, and

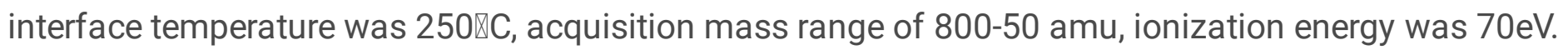
Helium is used as a carrier gas. Compounds were identified using a library search of the National Institute of Standards and Technology (NIST) database ${ }^{35}$, and mass spectral fragmentation patterns with those reported in the literature ${ }^{36}$.

\section{Test insects}

A. craccivora was collected from the open field conditions and maintained/reared on live French bean, Phaseolus vulgaris $\mathrm{L}$. under controlled conditions $(25 \pm 2 \rrbracket C$ temperature; $60 \pm 5 \%$ humidity and photoperiod 16:8 Light: Day) for more than 100 generations. The fresh nymphs of 3-4 days old were selected for experiments.

\section{Statement for confirmation of experimental protocols}

Experimental protocols are approved by the institutional committee (Publication committee) vide reference number 4903. However, we have not used any experimental animals for the present study.

\section{Preliminary screening}

Preliminary screening of leaf/bark extracts, fractions, seed oil, and compounds was carried out at higher concentrations (5000-10000 $\mathrm{mg} \mathrm{L}^{-1}$ ) for their efficacy against nymphs of $\boldsymbol{A}$. craccivora. Based on preliminary data, 5-6 concentrations were prepared and evaluated against aphid in the final bioassay experiments.

\section{Residual toxicity of leaf/bark aqueous ethanolic/methanolic extracts under laboratory}

Residual toxicity of leaf/bark extract/fractions and seed oil was tested against nymphs of $\boldsymbol{A}$. craccivora by the Potter spray method as per the standard method ${ }^{37}$. Briefly, five concentrations of LEE/LME/BEE/BME (500-20000 mg L-1), leaf and bark fractions (250-10000 mg L-1), SO (500-10000 mg $\mathrm{L}^{-1}$ ) and compounds (312.5-5000 $\mathrm{mg} \mathrm{L}^{-1}$ ) were prepared. The leaf discs of $3 \mathrm{~cm}$ diameter were prepared and pressed over the water-agar medium on Petri plates to maintain the freshness of leaf discs. About 10 nymphs/leaf disc were released in each Petri plate. Each treatment was replicated thrice. The test solution (2 ml) was sprayed under Potter's spray tower operated at $1.1 \mathrm{~kg} / \mathrm{cm}^{2}$ pressure and incubated under controlled conditions. Observations on dead insects were recorded at $24 \mathrm{~h}$ intervals and up to $96 \mathrm{~h}$. The commercial neem-based formulation i.e., Indo-neem (Azadirachtin 0.15\% EC and neem oil 35\%) used as a positive control for comparison. 


\section{Residual toxicity of binary mixtures of leaf/bark extracts and seed oil under laboratory}

Based on preliminary study, the blends/mixtures of seed oil with leaf /bark ethanol aqueous extract of $T$. sebifera was prepared in five concentrations $\left(62.5-1000 \mathrm{mg} \mathrm{L}^{-1}\right)$ and proportions as 1:3 (SO: LEE), 1:1 (SO: LEE), 3:1 (SO: LEE), 1:1:1 (SO: LEE: BEE), \{(1+1): 2$\}$ (LEE+SO: BEE), \{(1+1): 2$\}$ (LEE+BEE: SO) and $(1+1)$ : 2 (SO+BEE: LEE) for bioassayand synergistic activity against $\boldsymbol{A}$. craccivora under laboratory conditions. Five concentrations (62.5-1000 $\left.\mathrm{mg} \mathrm{L}^{-1}\right)$ were prepared and sprayed against nymphs of $\boldsymbol{A}$. craccivora as described above. Each treatment was replicated thrice. Observations on mortality were recorded at 24, 48, 72, and $96 \mathrm{~h}$ after treatment. The fractional effect indices (FEI) were calculated to study the joint action of binary mixtures/combinations. FEl=fractional effect of $A+$ fractional effect of $B$, in which fractional effect of $A=L C_{50}$ mixture /LC $C_{50}$ of $A$ and fractional effect of $B=L C_{50}$ mixture $/ L C_{50}$ of $\mathrm{B}^{38}$. The FEls were interpreted based on Bassole et al. ${ }^{39}$ classifications as being synergistic if $\mathrm{FEI}<0.5$, additive if $\mathrm{FEI} \geq 0.5$ and $\leq 1.0$, indifferent if $\mathrm{FEI}>1.0$ and $\leq 4.0$ or antagonistic if $\mathrm{FEI}>4.0$.

\section{Residual toxicity of leaf/bark extracts and its binary mixtures under plant growth chamber}

The residual toxicity of leaf/bark ethanolic aqueous extract, seed oil at six concentrations (625-20000 mg $\mathrm{L}^{-1}$ ) and its combinations of $\boldsymbol{T}$. sebifera (SO+LEE, SO+BEE) in a 1:1 ratio was prepared in five concentrations (125-2000 $\mathrm{mg} \mathrm{L}^{-1}$ ) and evaluated against $\boldsymbol{A}$. craccivora under controlled conditions (26 \pm $2 \varangle \mathrm{C}, 70 \pm 5 \%$ relative humidity and photoperiod of $16 \mathrm{~h}$ light and $8 \mathrm{~h}$ dark) in a plant growth chamber (Percival Scientific, Perry, lowa). The Phaseolus vulgaris plants were raised on plastic pots $(7 \times 9 \mathrm{~cm})$ filled with the potting mixture (1:1:1 ratio of vermiculate: cocopeat: perlite). One-week old plants (3-4 leaf stage) were inoculated with two days old nymphs of $\boldsymbol{A}$. craccivora and allowed to settle for $24 \mathrm{~h}$. After settling, test solutions/concentrations were sprayed on plants using a hand sprayer. Observations of the number of dead insects/plants were recorded at 24, 48, 72, and $96 \mathrm{~h}$ after treatment. There were six treatments and each treatment was replicated five times. The fractional effect indices (FEI) were calculated as mentioned above section to study the joint action of binary mixtures/combinations.

\section{Enzyme inhibition activities of LEE, BEE and SO against $A$. craccivora}

Four different concentrations of LEE, BEE and SO $(0.25,0.5 .1$ and $2 \%)$ were chosen for enzyme activity ${ }^{32}$ according to the residual toxicity assay as described above. The nymphs of $A$. craccivora of each concentration after $48 \mathrm{~h}$ of treatment were homogenised and centrifuged at $15000 \mathrm{rpm}$ at $4^{\circ} \mathrm{C}$ for 30 minutes. The pellet was discarded and the supernatant was used for the enzyme activity. Before proceeding with enzyme activity, the total protein concentration was measured using the Bradford reagent. For the enzyme assay of glutathione S-transferase (GST) and acetylcholine esterase (AChE), the assay kits were procured from Cayman Chemicals and Abcam, respectively.

\section{Data analysis}

The mortality data of aphids based on residual toxicity of extracts, fractions, seed oil, combinations, and pure compounds were compiled. The median lethal concentration values $\left(\mathrm{LC}_{50}\right)$ and other regression 
parameters were determined by Probit ${ }^{40}$ using SPSS 10 software, version 16 . The FEl for binary mixtures/combinations were calculated to study the joint action studies (synergistic, additive, indifferent, and antagonistic). Similarly, percent mortality data against aphids were also analysed by one-way analysis of variance, and means were compared by Tukey's post hoc test.

\section{Results}

Fatty acid composition of seed oil of T. sebifera

The fatty acid composition and distribution of $T$. sebifera are presented in Table 1 . The SO of $T$. sebifera gives a yield of $50 \%(\mathrm{v} / \mathrm{w})$. The saturated fatty acids are present in the SO were palmitic acid $(4.99 \%)$ and stearic acid (1.38\%.). The unsaturated fatty acids are oleic acid (8.78\%), linoleic acid $(15.42 \%)$, and palmitoleic acid $(2.13 \%)$. The distribution of saturated fatty acids is less $(6.73 \%)$ as compared to unsaturated fatty acids $(26.33 \%)$.

Table 1

Chemical composition of fatty acid methyl esters present in seed oil of Triadica sebifera; Note: $\mathrm{RI}^{\mathrm{a}}=$ Calculated Retention Indices; $\mathrm{RI}^{\mathrm{b}}=$ Retention Indices from literature

\begin{tabular}{|c|c|c|c|c|c|}
\hline $\begin{array}{l}\text { Sr. } \\
\text { No. }\end{array}$ & Compound's name & $\begin{array}{l}\text { Area } \\
(\%)\end{array}$ & $\mathrm{R}^{\mathrm{a}}$ & $\mathrm{RI}^{\mathrm{b}}$ & Mass fragmentation \\
\hline 1 & $\begin{array}{l}\text { 9-Hexadecenoic acid, methyl ester } \\
\text { (Palmitoleic acid, } \mathrm{C}_{6}: 1 \text { ) }\end{array}$ & 2.13 & 1933 & $1931^{41}$ & $\begin{array}{l}268\left[\mathrm{M}^{+}\right], 236,207,194 \\
152,138,123,97,74,69 \\
55,41\end{array}$ \\
\hline 2 & $\begin{array}{l}\text { Hexadecanoic acid, methyl ester } \\
\left(\text { Palmitic acid, } \mathrm{C}_{16}: 0\right)\end{array}$ & 4.99 & 1986 & $1984^{42}$ & $\begin{array}{l}270\left[\mathrm{M}^{+}\right], 239,227,199 \\
185,171,143,129,101 \\
87,74,57,41\end{array}$ \\
\hline 3 & $\begin{array}{l}\text { 9,12-Octadecadienoic acid (Z, Z), } \\
\text { methyl ester (Linoleic acid, } C_{18}: 2 \text { ) }\end{array}$ & 15.42 & 2092 & $2094^{43}$ & $\begin{array}{l}294\left[\mathrm{M}^{+}\right], 262,178,164 \\
150,135,123,109,95 \\
81,67,55,41\end{array}$ \\
\hline 4 & $\begin{array}{l}\text { 9-Octadecenoic acid, methyl ester } \\
\text { (Oleic acid, } \mathrm{C}_{18}: 1 \text { ) }\end{array}$ & 8.78 & 2100 & $2101^{44}$ & $\begin{array}{l}296\left[\mathrm{M}^{+}\right], 264,222,180 \\
166,152,137,123,97 \\
83,69,55,41\end{array}$ \\
\hline 5 & $\begin{array}{l}\text { Octadecanoic acid, methyl ester } \\
\text { (Stearic acid, } \mathrm{C}_{18}: 0 \text { ) }\end{array}$ & 1.38 & 2121 & $2128^{45}$ & $\begin{array}{l}298\left[\mathrm{M}^{+}\right], 255,213,199, \\
143,129,101,87,74,57, \\
43,41\end{array}$ \\
\hline
\end{tabular}

\section{Structure Elucidation Of Isolated Compounds}

Compound 1 was isolated as a white amorphous powder. The ESI-QTOF-MS of the compound showing the molecular ion peak at $\mathrm{m} / \mathrm{z} 471.08(\mathrm{M}+\mathrm{Na})^{+}$indicated the molecular formula as $\mathrm{C}_{21} \mathrm{H}_{20} \mathrm{O}_{11}$ (Fig. S1). Aromatic signals observed in proton spectrum at $\delta_{\mathrm{H}} 7.98 \mathrm{~d}(2 \mathrm{H}, \mathrm{J}=8.4 \mathrm{~Hz}), 6.81 \mathrm{~d}(2 \mathrm{H}, \mathrm{J}=8.4 \mathrm{~Hz}), 6.30 \mathrm{~s}$ $(1 \mathrm{H})$, and $6.11 \mathrm{~s}(1 \mathrm{H})$ showing the presence of two sets of equivalent protons and two singlet protons 
which confirms the presence of two aromatic rings in the structure. Also, the presence of a doublet signal at $\delta_{H} 5.16 \mathrm{~d}(1 \mathrm{H}, J=7.2 \mathrm{~Hz})$ which showing the HSQC correlation with $\delta c 104.2$ indicates the presence of a $\beta$-D-glucose moiety, which was also confirmed by COSY and HMBC correlations. The carbon value at $\delta c$ 179.4 showed the presence of the $\mathrm{C}=0$ group. Hence from the above spectral data and comparison with literature data ${ }^{12}$, compound 1 was identified as Kaempferol-3-0-glucoside (Fig. S2).

Kaempferol-3-0-glucoside: White amorphous powder; 128 mg; mp. $178^{\circ} \mathrm{C}$; ESI-QTOF-MS (Positive) $\mathrm{m} / \mathrm{z} .471 .08(\mathrm{M}+\mathrm{Na}){ }^{+1} \mathrm{H}-\mathrm{NMR}\left(600 \mathrm{MHz}, \mathrm{CD}_{3} \mathrm{OD}\right)$ (Fig. S3 and Table S1), ${ }^{13} \mathrm{C}-\mathrm{NMR}\left(150 \mathrm{MHz}, \mathrm{CD}_{3} \mathrm{OD}\right)$ (Fig. S4 and Table S1).

Compound 2 was isolated as a white amorphous powder. The molecular formula $\mathrm{C}_{21} \mathrm{H}_{20} \mathrm{O}_{11}$ was calculated after ESI-QTOF-MS, which was showing the molecular ion peak at $m / z 487.08\left(\mathrm{M}^{\left.-\mathrm{Na}^{+}\right)}(\mathrm{Fig}\right.$. S5). Compound 2 was identified by comparing observed data with literature-reported data ${ }^{13}$.After comparison of observed data with literature, compound 2 was identified as Quercetin-3-0-glucoside (Fig. S2).

Quercetin-3-0-glucoside: White amorphous powder; $150 \mathrm{mg}$; mp. $226^{\circ} \mathrm{C}$; ESI-QTOF-MS (Positive) $\mathrm{m} / \mathrm{z} .487 .08\left(\mathrm{M}^{\mathrm{N}} \mathrm{Na}^{+}\right) .{ }^{1} \mathrm{H}-\mathrm{NMR}\left(600 \mathrm{MHz}, \mathrm{CD}_{3} \mathrm{OD}\right)$ (Fig. S6 and Table S1), ${ }^{13} \mathrm{C}-\mathrm{NMR}\left(150 \mathrm{MHz}, \mathrm{CD}_{3} \mathrm{OD}\right)$ (Fig. S7 and Table S1).

Compound $\mathbf{3}$ was obtained as a white powder. The molecular ion peak was observed at $m / z 341.03$ $(2 \mathrm{M}+\mathrm{H})^{+}$in ESI-QTOF-MS (Fig. S8), which indicates the formation of a non-covalent dimer in ESI$\mathrm{MS}^{14}$. Hence the stable molecular ion peak was formed in the form of a non-covalent dimer $(2 \mathrm{M}+\mathrm{H})^{+}$. From spectral analysis and literature reports ${ }^{15}$, compound $\mathbf{3}$ was identified as gallic acid (Fig. S2).

Gallic acid: White powder; $124 \mathrm{mg}$; mp. $260^{\circ} \mathrm{C}$; ESI-QTOF-MS (Positive) $\mathrm{m} / \mathrm{z} .341 .03(2 \mathrm{M}+\mathrm{H})^{+} ;{ }^{1} \mathrm{H}-\mathrm{NMR}$ $\left(600 \mathrm{MHz}, \mathrm{CD}_{3} \mathrm{OD}\right.$ ) (Fig. S9 and Table S2), ${ }^{13} \mathrm{C}-\mathrm{NMR}\left(150 \mathrm{MHz}, \mathrm{CD}_{3} \mathrm{OD}\right)$ (Fig. S10 and Table S2).

Similarly, compound $\mathbf{4}$ was also isolated as a white powder. The molecular ion peak for compound $\mathbf{4}$ was obtained at $m / z 349.32(2 \mathrm{M}+\mathrm{H})^{+}($Fig. S11), which was also the molecular ion peak of the non-covalent dimer peak of compound $\mathbf{4}$. ESI-QTOF-MS analyses of compound $\mathbf{4}$ were indicated its molecular formula $\mathrm{C}_{7} \mathrm{H}_{10} \mathrm{O}_{5}$ and after analysis and comparison of observed data with literature ${ }^{16}$, compound $\mathbf{4}$ was identified as Shikimic acid (Fig. S2).

Shikimic acid: White powder; $108 \mathrm{mg}$; mp. $184-185^{\circ} \mathrm{C}$, ESI-QTOF-MS (Positive) $\mathrm{m} / \mathrm{z}$. $349.32(2 \mathrm{M}+\mathrm{H})^{+} ;{ }^{1} \mathrm{H}-$ NMR (600 MHz, CD 3 OD) (Fig. S12 and Table S2), ${ }^{13}$ C-NMR (150 MHz, CD ${ }_{3} \mathrm{OD}$ ) (Fig. S13 and Table S2).

\section{Gas chromatography-mass spectrophotometry (GC-MS) analysis of n-hexane fractions}

The metabolites and their mass fragmentation, identified through GC-MS in the $n$-hexane fractions of leaf and bark were presented in Table 2. In the $n$-hexane fraction of the leaf, the major metabolites were $n$ - 
Hexadecanoic acid (15.61\%) and were followed by octadecanoic acid, ethyl ester (9.85\%) and neophytadiene (5.87\%). In the case of the $n$-hexane fraction of the bark, the major metabolites were galaxolide $(44.73 \%)$ and were followed by ethyl phthalate $(28.43 \%)$ and 1 -Octadecene $(2.69 \%)$.

Table 2

Chemical composition of $n$-hexane fraction from leaf and bark of Triadica sebifera; Note: $\mathrm{RI}^{\mathrm{a}}=$ Calculated Retention Indices; $\mathrm{RI}^{\mathrm{b}}=$ Retention Indices from literature

\begin{tabular}{|c|c|c|c|c|c|}
\hline Metabolites & $\begin{array}{l}\text { Molecular } \\
\text { formula }\end{array}$ & $\begin{array}{l}\text { Area } \\
(\%)\end{array}$ & $\mathrm{R}^{\mathrm{a}}$ & $\mathrm{RI}^{\mathrm{b}}$ & Mass fragmentation \\
\hline \multicolumn{6}{|l|}{ Leaf } \\
\hline 1,8-Cineole & $\mathrm{C}_{10} \mathrm{H}_{18} \mathrm{O}$ & 1.61 & 1037 & $1037^{46}$ & $\begin{array}{l}m / z 154\left(M^{+}\right), 139,125,108,84,81 \\
69,43,41\end{array}$ \\
\hline Fenchyl acetate & $\mathrm{C}_{12} \mathrm{H}_{20} \mathrm{O}_{2}$ & 0.70 & 1230 & $1223^{47}$ & $\begin{array}{l}m / z 154\left(M^{+}\right), 136,121,108,95,80 \\
69,43,41,27\end{array}$ \\
\hline Neophytadiene & $\mathrm{C}_{20} \mathrm{H}_{38}$ & 5.87 & 1841 & $1836^{48}$ & $\begin{array}{l}m / z 137\left(\mathrm{M}^{+}\right), 123,109,95,82,68 \\
43,41\end{array}$ \\
\hline $\begin{array}{l}n \text { Hexadecanoic } \\
\text { acid }\end{array}$ & $\mathrm{C}_{16} \mathrm{H}_{32} \mathrm{O}_{2}$ & 15.61 & 1961 & $1962^{49}$ & $\begin{array}{l}m / z 256\left(\mathrm{M}^{+}\right), 227,213,199,185 \\
171,157,143,129,115,98,85,73 \\
60,43,41\end{array}$ \\
\hline $\begin{array}{l}\text { cis, cis, cis-7,10,13- } \\
\text { Hexadecatrienal }\end{array}$ & $\mathrm{C}_{16} \mathrm{H}_{26} \mathrm{O}$ & 3.06 & 1992 & $1989^{50}$ & $\begin{array}{l}m / z 264\left(\mathrm{M}^{+}\right), 222,149,135,121 \\
108,95,79,67,55,41\end{array}$ \\
\hline $\begin{array}{l}\text { Octadecanoic acid, } \\
\text { ethyl ester }\end{array}$ & $\mathrm{C}_{20} \mathrm{H}_{40} \mathrm{O}_{2}$ & 9.85 & 2189 & $2189^{51}$ & $\begin{array}{l}m / z 312\left(M^{+}\right), 269,213,157,143 \\
129,115,101,88,70,57,43,41\end{array}$ \\
\hline \multicolumn{6}{|l|}{ Bark } \\
\hline Hexanoic acid & $\mathrm{C}_{6} \mathrm{H}_{12} \mathrm{O}_{2}$ & 0.31 & 984 & $989^{52}$ & $m / z 87\left(M^{+}\right), 73,60,43,41,40$ \\
\hline 2-Decenal & $\mathrm{C}_{10} \mathrm{H}_{18} \mathrm{O}$ & 0.23 & 1263 & $1260^{53}$ & $\begin{array}{l}m / z 121\left(\mathrm{M}^{+}\right), 98,84,70,57,43,41 \\
\text {, }\end{array}$ \\
\hline Ethyl phthalate & $\mathrm{C}_{12} \mathrm{H}_{17} \mathrm{O}_{4}$ & 28.43 & 1587 & $1585^{54}$ & $\begin{array}{l}m / z 222\left(M^{+}\right), 177,149,121,105,93, \\
76,65,50\end{array}$ \\
\hline 1-Octadecene & $\mathrm{C}_{18} \mathrm{H}_{36}$ & 2.69 & 1803 & $1800^{55}$ & $m / z 125\left(M^{+}\right), 111,97,83,57,41,40$ \\
\hline Galaxolide & $\mathrm{C}_{18} \mathrm{H}_{26} \mathrm{O}$ & 44.73 & 1834 & $1837^{56}$ & $\begin{array}{l}m / z 258\left(M^{+}\right), 243,213,185,171 \\
157,143,128\end{array}$ \\
\hline
\end{tabular}

Residual toxicity of leaf/bark extracts and seed oil of T. sebifera against A. craccivora

The residual toxicity of $L E E, L M E, B E E, B M E$ and $S O$ of $T$. sebifera against aphid with respect toLC $C_{50}$ values are presented in Table 3. Bark extracts are most effective than leaf extracts. Among leaf extracts, 
LEE $80 \%$ was found most effective $\left(\mathrm{LC}_{50}=9590.49 \mathrm{mg} \mathrm{L}^{-1}\right)$ against $A$. craccivora after $72 \mathrm{~h}$ and was followed by $L M E 100 \%, 80 \%$, and $50 \%\left(L_{50}=9627,10800\right.$, and $11540 \mathrm{mg} \mathrm{L}^{-1}$, respectively) as compared to LEE $100 \%\left(\mathrm{LC}_{50}=14100 \mathrm{mg} \mathrm{L}^{-1}\right)$. Similarly, $96 \mathrm{~h}$ after treatment also, LEE $80 \%$ was found more effective $\left(\mathrm{LC}_{50}=6756.42 \mathrm{mg} \mathrm{L}^{-1}\right)$ and was followed by LME 80 and $100 \%\left(\mathrm{LC}_{50}=7120.27\right.$ and $7528.56 \mathrm{mg}$ $\mathrm{L}^{-1}$, respectively) as compared to $\mathrm{LME} 50 \%$ and LEE $100 \%\left(\mathrm{LC}_{50}=7579.55\right.$ and $8702.07 \mathrm{mg} \mathrm{L}^{-1}$, respectively). 
Table 3

Efficacy of ethanolic/methanolic leaf/bark aqueous extracts and seed oil of Triadica sebifera against Aphis craccivora; Note: $\mathrm{LC}_{50}=$ Lethal concentration to kill $50 \%$ of test insect; Mean of three replications; $n=150$ insects per treatment; $L_{50}$ was calculated for fractions showing $>50 \%$ mortality using Probit analysis

\begin{tabular}{|c|c|c|c|c|c|}
\hline Leaf extracts & $\begin{array}{l}\mathrm{LC}_{50} \\
\left(\mathrm{mg} \mathrm{L}^{-1}\right)\end{array}$ & $\begin{array}{l}\text { Confidence limits } \\
\left(\mathrm{mg} \mathrm{L}^{-1}\right)\end{array}$ & Slope \pm SE & Chi square & $P$ value \\
\hline LEE 100\% (72 h) & 14100.0 & $11764.41-17527.10$ & $2.31 \pm 0.53$ & 0.36 & 0.99 \\
\hline LEE 100\% (96 h) & 8702.07 & $6880.35-10137.00$ & $3.08 \pm 0.52$ & 0.61 & 0.99 \\
\hline LEE 80\% (72 h) & 9590.49 & $7706.54-11128.43$ & $2.91 \pm 0.52$ & 1.81 & 0.87 \\
\hline LEE 80\% (96 h) & 6756.42 & $5342.84-7885.95$ & $3.97 \pm 0.58$ & 1.84 & 0.87 \\
\hline LEE 50\% (72 h) & 38860.0 & $24892.46-341196.32$ & $2.07 \pm 0.72$ & 0.67 & 0.98 \\
\hline LEE 50\% (96 h) & 28570.0 & $21383.79-71689.83$ & $2.51 \pm 0.71$ & 0.68 & 0.98 \\
\hline LME 100\% (72 h) & 9627.0 & $6881.53-11725.61$ & $2.07 \pm 0.49$ & 2.02 & 0.85 \\
\hline LME 100\% (96 h) & 7528.56 & $5691.95-8931.12$ & $3.04 \pm 0.52$ & 5.07 & 0.41 \\
\hline LME 80\% (72 h) & 10800.0 & $8603.89-12754.79$ & $2.45 \pm 0.50$ & 2.24 & 0.81 \\
\hline LME 80\% (96 h) & 7120.27 & $5593.04-8326.45$ & $3.63 \pm 0.55$ & 6.95 & 0.22 \\
\hline LME 50\% (72 h) & 11540.0 & $9689.45-13331.76$ & $2.86 \pm 0.53$ & 3.76 & 0.58 \\
\hline LME 50\% (96 h) & 7579.55 & $6239.59-8676.68$ & $4.15 \pm 0.58$ & 5.80 & 0.33 \\
\hline Seed oil (72 h) & 2504.59 & $1675.92-3562.91$ & $1.18 \pm 0.21$ & 0.86 & 0.97 \\
\hline Seed oil (96 h) & 850.938 & $533.52-1171.05$ & $1.69 \pm 0.25$ & 3.28 & 0.66 \\
\hline \multicolumn{6}{|l|}{ Bark extracts } \\
\hline BEE 100\% (72 h) & 8325.46 & $6958.41-9455.76$ & $4.05 \pm 0.57$ & 7.39 & 0.19 \\
\hline BEE 100\% (96 h) & 5228.89 & $4038.43-6165.80$ & $4.88 \pm 0.78$ & 3.83 & 0.57 \\
\hline BEE 80\% (72 h) & 7300.57 & $5889.44-8435.86$ & $3.97 \pm 0.57$ & 6.17 & 0.29 \\
\hline BEE 80\% (96 h) & 5115.98 & $3613.44-6219.77$ & $4.04 \pm 0.75$ & 1.31 & 0.73 \\
\hline BEE 50\% (72 h) & 10650.0 & $8244.02-12743.89$ & $2.25 \pm 0.50$ & 3.73 & 0.59 \\
\hline BEE 50\% (96 h) & 7098.41 & $5159.65-8546.32$ & $2.91 \pm 0.52$ & 7.32 & 0.20 \\
\hline BME 100\% (72 h) & 8737.64 & $6586.26-10377.96$ & $2.61 \pm 0.50$ & 2.12 & 0.83 \\
\hline BME 100\% (96 h) & 5701.69 & $4147.42-6880.23$ & $3.73 \pm 0.63$ & 2.34 & 0.67 \\
\hline BME 80\% (72 h) & 9490.58 & $7504.78-11085.89$ & $2.78 \pm 0.51$ & 0.45 & 0.99 \\
\hline
\end{tabular}




\begin{tabular}{|llllll|}
\hline Leaf extracts & $\begin{array}{l}\mathrm{LC}_{50} \\
\left(\mathrm{mg} \mathrm{L}^{-1}\right)\end{array}$ & $\begin{array}{l}\text { Confidence limits } \\
\left(\mathrm{mg} \mathrm{L}^{-1}\right)\end{array}$ & Slope \pm SE & Chi square & P value \\
\hline BME 80\% $(96 \mathrm{~h})$ & 5779.72 & $4187.11-7007.96$ & $3.55 \pm 0.58$ & 1.42 & 0.92 \\
\hline BME 50\% $(72 \mathrm{~h})$ & 10580.0 & $8431.88-12452.44$ & $2.51 \pm 0.51$ & 1.43 & 0.92 \\
\hline BME 50\% $(96 \mathrm{~h})$ & 5233.81 & $2941.71-6869.58$ & $2.51 \pm 0.53$ & 7.29 & 0.20 \\
\hline Azadirachtin $(72 \mathrm{~h})$ & 2642.32 & $2013.70-3816.64$ & $1.53 \pm 0.22$ & 0.99 & 0.80 \\
\hline Azadirachtin $(96 \mathrm{~h})$ & 1174.22 & $973.61-1416.60$ & $2.28 \pm 0.25$ & 5.16 & 0.16 \\
\hline
\end{tabular}

Among bark extracts, $B E E 80 \%\left(L_{50}=7300.57 \mathrm{mg} \mathrm{L}^{-1}\right)$ was most effective against aphid after $72 \mathrm{~h}$, then $\mathrm{BEE}$ and $\mathrm{BME} 100 \%\left(\mathrm{LC}_{50}=8325.46\right.$ and $8737.64 \mathrm{mg} \mathrm{L}^{-1}$, respectively) as compared to $\mathrm{BME} 80 \%, 50 \%$ and BEE 50\% ( $\mathrm{LC}_{50}=9490.58,10580$ and $10650 \mathrm{mg} \mathrm{L}^{-1}$, respectively). Similarly, $96 \mathrm{~h}$ after treatment, $\mathrm{BEE}$ $80 \%\left(\mathrm{LC}_{50}=5115.98 \mathrm{mg} \mathrm{L}^{-1}\right)$ was found to be more effective and was followed by BEE $100 \%$ $\left(\mathrm{LC}_{50}=5228.89 \mathrm{mg} \mathrm{L}^{-1}\right)$ as compared to BME 50\%, 100\%, 80\%, and BEE 50\% $\left(\mathrm{LC}_{50}=5233.81,5701.69\right.$, 5779.72 and $7098.41 \mathrm{mg} \mathrm{L}^{-1}$, respectively).

The SO of T. sebifera in the current study was found to be more effective after $72 \mathrm{~h}$ and $96 \mathrm{~h}$ of treatment $\left(\mathrm{LC}_{50}=2504.59\right.$ and $850.94 \mathrm{mg} \mathrm{L}^{-1}$, respectively) than BEE and $\mathrm{LEE}\left(\mathrm{LC}_{50}=5115.98-6756.42 \mathrm{mg} \mathrm{L}^{-1}\right)$. All the leaf and bark extracts were not superior to the positive control, Indo-neem (Azadirachtin $0.15 \% \mathrm{EC}$ ) after $72 \mathrm{~h}$ and $96 \mathrm{~h}\left(\mathrm{LC}_{50}=2642.32\right.$ and $1174.22 \mathrm{mg} \mathrm{L}^{-1}$, respectively). However, the SO is more superior to leaf and bark extracts.

\section{Residual toxicity of thecombination of seed oil with leaf/bark extract of T. sebifera and itssynergistic activity against $A$. craccivora under laboratory}

The residual toxicity of thecombination of SO with 80\% LEE/BEE and its synergistic activity of $T$. sebifera against $A$. craccivoraunderlaboratory conditions were presented in Table S3-S4. Results showed that among combinations studied, a mixture of LEE $80 \%+$ SO and BEE $80 \%\{(1+1): 2\}$ was found more effective $\left(\mathrm{LC}_{50}=239.94 \mathrm{mg} \mathrm{L}^{-1}\right)$ against nymphs of $A$. craccivora after $72 \mathrm{~h}$ and was followed by a mixture of $B E E+S O(1: 1)\left(L C_{50}=263.56 \mathrm{mg} \mathrm{L}^{-1}\right), S O+L E E$ at $1: 1$ ratio $\left(L_{50}=303.29 \mathrm{mg} \mathrm{L}^{-1}\right)$ and $\mathrm{LEE}+\mathrm{BEE}+\mathrm{SO}$ at 1:1:1 ratio $\left(\mathrm{LC}_{50}=337.33 \mathrm{mg} \mathrm{L}^{-1}\right)$ as compared to other mixtures/blends. Based on the fractional effect index $(\mathrm{FEI})$, all the combinations/blends evaluated against $A$. craccivora showed synergistic interaction after $72 \mathrm{~h}$. Among them, LEE+BEE at 1:3, 3:1, and 1:1 ratio showed the most significant synergistic interaction ( $\mathrm{FEI}=0.088,0.092$ and 0.122 , respectively), as compared to other blends/combinations. Similarly, $96 \mathrm{~h}$ after treatment, BEE + SO (1:1 ratio) was found to be more effective $\left(\mathrm{LC}_{50}=144.26 \mathrm{mg} \mathrm{L}^{-1}\right)$ against $A$. craccivoraand was followed by SO+LEE at 1:1 ratio $\left(\mathrm{LC}_{50}=168.9 \mathrm{mg}\right.$ $\left.\mathrm{L}^{-1}\right), \mathrm{LEE}+\mathrm{SO}+\mathrm{BEE}$ at $\{(1+1): 2\}$ ratio $\left(\mathrm{LC}_{50}=170.46 \mathrm{mg} \mathrm{L}^{-1}\right)$, $\mathrm{LEE}+\mathrm{BEE}$ at $1: 3$ ratio $\left(\mathrm{LC}_{50}=179.31 \mathrm{mg} \mathrm{L}^{-1}\right)$ as compared to other mixtures/blends. Based on FEl, all the combinations/blends evaluated against $A$. 
craccivora showed a synergistic effect. Among them, LEE+BEE at 1:3, 3:1, and 1:1 ratio showed significant synergistic interaction ( $\mathrm{FEI}=0.061,0.070$, and 0.100 , respectively), as compared to other blends/mixtures.

Residual toxicity of seed oil, leaf/bark extracts and its binary mixtures of T. sebifera and itssynergistic activity against $A$. craccivoraunder plant growth chamber

Residual toxicity of seed oil, leaf/bark extracts, and its binary mixtures (1:1) of $T$. sebiferaagainst $A$. craccivoraunder plant growth chamber conditions are presented in Table S5.Results showed that the binary mixture of SO+LEE (1:1 ratio) was found to be more effective against $A$. craccivora $\left(\mathrm{LC}_{50}=264.05\right.$ $\left.\mathrm{mg} \mathrm{L}^{-1}\right)$ after $72 \mathrm{~h}$ and was followed by SO+BEE $\left(\mathrm{LC}_{50}=362.76 \mathrm{mg} \mathrm{L}^{-1}\right)$ as compared to SO, LEE, and BEE $\left(\mathrm{LC}_{50}=1100.22,1840.84\right.$ and $5073.99 \mathrm{mg} \mathrm{L}^{-1}$, respectively). Based on FEl values, both the blends (SO+LEE and $\mathrm{SO}+\mathrm{BEE}$ ) showed synergistic interaction ( $\mathrm{FEI}=0.38$ and 0.40 ) against $A$. craccivora (Table S5). Similarly, $96 \mathrm{~h}$ after treatment, binary mixtures of SO+LEE was were more effective against $A$. craccivora $\left(\mathrm{LC}_{50}=223.82 \mathrm{mg} \mathrm{L}^{-1}\right)$ and was followed by SO+BEE $\left(\mathrm{LC}_{50}=247.54 \mathrm{mg} \mathrm{L}^{-1}\right)$ as compared to $\mathrm{LEE}, \mathrm{SO}$, and $\mathrm{BEE}\left(\mathrm{LC}_{50}=685.47,706.53\right.$ and $2328.79 \mathrm{mg} \mathrm{L}^{-1}$, respectively). Based on FEl values, $\mathrm{SO}+\mathrm{BEE}$ showed synergistic interaction $(\mathrm{FEI}=0.46)$. A binary mixture of $\mathrm{SO}+\mathrm{LEE}$ showed additive interaction after $96 \mathrm{~h}$ of treatment.

Residual toxicity of leaf and bark fractions of T. sebifera against A. craccivora

The residual toxicity of leaf/bark fractions of $T$. sebifera against $A$. craccivora was presented in Table 4. Results showed that, among leaf fractions, $n$-hexane fraction $\left(\mathrm{LC}_{50}=425.73 \mathrm{mg} \mathrm{L}^{-1}\right)$ was more effective than ethyl acetate and $n$-butanol $\left(\mathrm{LC}_{50}=838.89\right.$ and $1527.84 \mathrm{mg} \mathrm{L}^{-1}$, respectively) as compared to water fraction $\left(\mathrm{LC}_{50}=2702.82 \mathrm{mg} \mathrm{L}^{-1}\right)$ after $72 \mathrm{~h}$. Similarly, $96 \mathrm{~h}$ after treatment, $n$-hexane fraction $\left(\mathrm{LC}_{50}=196.61 \mathrm{mg} \mathrm{L}^{-1}\right)$ was the most promising efficacy than ethyl acetate $\left(\mathrm{LC}_{50}=367.75 \mathrm{mg} \mathrm{L}^{-1}\right)$ as compared to water and $n$-butanol fraction ( $\mathrm{LC}_{50}=864.68$ and $1527.84 \mathrm{mg} \mathrm{L}^{-1}$, respectively). 
Table 4

Efficacy of fractions of Triadica sebifera leaf and bark against Aphis craccivora; Note: $\mathrm{LC}_{50}=$ Lethal concentration to kill $50 \%$ of test insect; Mean of three replications; $n=150$ insects per treatment; $L_{50}$ was calculated for fractions showing $>50 \%$ mortality using Probit analysis

\begin{tabular}{|c|c|c|c|c|c|}
\hline Leaf fractions & $\begin{array}{l}\mathrm{LC}_{50} \\
\left(\mathrm{mg} \mathrm{L}^{-1}\right)\end{array}$ & Confidence limits $\left(\mathrm{mg} \mathrm{L}^{-1}\right)$ & Slope \pm SE & Chi square & $P$ value \\
\hline$n$-hexane (72 h) & 425.73 & $196.38-679.40$ & $1.09 \pm 0.21$ & 1.55 & 0.82 \\
\hline$n$-hexane (96 h) & 196.61 & $76.21-316.54$ & $1.57 \pm 0.33$ & 2.45 & 0.65 \\
\hline Ethyl acetate (72 h) & 838.89 & $558.64-1178.77$ & $1.39 \pm 0.21$ & 3.96 & 0.41 \\
\hline Ethyl acetate (96 h) & 367.75 & $230.65-503.57$ & $1.88 \pm 0.32$ & 0.75 & 0.94 \\
\hline$n$-butanol (72 h) & 1527.84 & $1123.04-2093.81$ & $1.60 \pm 0.22$ & 1.14 & 0.89 \\
\hline$n$-butanol (96 h) & 990.22 & $746.43-1294.96$ & $1.92 \pm 0.25$ & 2.68 & 0.61 \\
\hline Water (72 h) & 2702.82 & $1799.12-4556.10$ & $1.12 \pm 0.19$ & 1.34 & 0.85 \\
\hline Water (96 h) & 864.68 & $643.83-1133.25$ & $1.89 \pm 0.25$ & 2.07 & 0.72 \\
\hline \multicolumn{6}{|l|}{ Bark fractions } \\
\hline$n$-hexane (72 h) & 1659.98 & $1211.70-2310.56$ & $1.54 \pm 0.21$ & 3.62 & 0.46 \\
\hline n-hexane (96 h) & 1130.95 & $867.74-1467.86$ & $2.02 \pm 0.26$ & 1.95 & 0.75 \\
\hline Ethyl acetate (72 h) & 3629.52 & $2322.33-6984.03$ & $1.05 \pm 0.19$ & 3.60 & 0.46 \\
\hline Ethyl acetate (96 h) & 813.45 & $613.57-1052.33$ & $2.04 \pm 0.27$ & 0.80 & 0.94 \\
\hline n-butanol (72 h) & 3539.63 & $2343.17-6296.58$ & $1.15 \pm 0.20$ & 1.92 & 0.75 \\
\hline n-butanol (96 h) & 1071.81 & $762.69-1472.81$ & $1.52 \pm 0.22$ & 5.26 & 0.26 \\
\hline Water $72 \mathrm{~h}$ ) & 3049.50 & $2087.70-4995.85$ & $1.24 \pm 0.20$ & 5.94 & 0.20 \\
\hline Water (96 h) & 915.15 & $684.74-1198.41$ & $1.90 \pm 0.25$ & 2.55 & 0.63 \\
\hline Azadirachtin (72 h) & 2642.32 & $2013.70-3816.64$ & $1.53 \pm 0.22$ & 0.99 & 0.80 \\
\hline Azadirachtin (96 h) & 1174.22 & $973.61-1416.60$ & $2.28 \pm 0.25$ & 5.16 & 0.16 \\
\hline
\end{tabular}

Among bark fractions, $n$-hexane fraction $\left(\mathrm{LC}_{50}=1659.98 \mathrm{mg} \mathrm{L}^{-1}\right)$ was most promising against $A$. craccivora after $72 \mathrm{~h}$ than water $\left(\mathrm{LC}_{50}=3049.50 \mathrm{mg} \mathrm{L}^{-1}\right)$ as compared to $n$-butanol and ethyl acetate $\left(\mathrm{LC}_{50}=3539.63\right.$ and $3629.52 \mathrm{mg} \mathrm{L}^{-1}$, respectively). Similarly, $96 \mathrm{~h}$ after treatment, ethyl acetate fraction was most effective $\left(\mathrm{LC}_{50}=813.45 \mathrm{mg} \mathrm{L}^{-1}\right)$ than water $\left(\mathrm{LC}_{50}=915.15 \mathrm{mg} \mathrm{L}^{-1}\right)$ as compared to $n$-butanol and $n$-hexane $\left(\mathrm{LC}_{50}=1071.81\right.$ and $1130.95 \mathrm{mg} \mathrm{L}^{-1}$, respectively). All the leaf and bark fractions are more 
superior than the positive control, Indo-neem (Azadirachtin 0.15\% EC) after 72 and $96 \mathrm{~h}\left(\mathrm{LC}_{50}=2642.32\right.$ and $1174.22 \mathrm{mg} \mathrm{L}^{-1}$, respectively).

Residual toxicity of isolated compounds of T. sebifera against A. craccivora

The experimental results on residual toxicity of the isolated compounds against $A$. craccivora with respect to $L C_{50}$ values are shown in Table 5.Among four compounds isolated from leaf fractions of $T$. sebifera, gallic acid was most effective $\left(\mathrm{LC}_{50}=1303.68 \mathrm{mg} \mathrm{L}^{-1}\right)$ against $A$. craccivora and was followed by shikimic acid and quercetin-3-0-glucoside $\left(\mathrm{LC}_{50}=1725.09-1855.93 \mathrm{mg} \mathrm{L}^{-1}\right)$ as compared to kaempferol-3O-glucoside $\left(\mathrm{LC}_{50}=3762.69 \mathrm{mg} \mathrm{L}^{-1}\right)$. With respect to per cent mortality, gallic acid at $5000 \mathrm{mg} \mathrm{L}^{-1}$ also reported $98 \%$ mortality $\left(F_{4,49}=135.79 ; p<0.0001\right)$ and was followed by shikimic acid, quercetin-3-0glucoside and kaempferol-3-0-glucoside ( 88,80 and 58\%, respectively) $\left(F_{4,49}=129.71,106.32\right.$ and 61.00; $(\star \star \star P<0.0001)$ (Fig. S14). All the tested compounds are not superior to the positive control, Indo-neem (Azadirachtin $0.15 \% \mathrm{EC}$ ) after $72 \mathrm{~h}$ and $96 \mathrm{~h}\left(\mathrm{LC}_{50}=2642.32\right.$ and $1174.22 \mathrm{mg} \mathrm{L}^{-1}$, respectively) except gallic acid after $72 \mathrm{~h}\left(\mathrm{LC}_{50}=2339.69 \mathrm{mg} \mathrm{L}^{-1}\right)$. 
Table 5

Efficacy of compounds isolated from leaf fractions of Triadica sebifera against Aphis craccivora; Note: $\mathrm{LC}_{50}=$ Lethal concentration to kill $50 \%$ of test insect; Mean of five replications; $\mathrm{n}=300$ insects per treatment; $\mathrm{LC}_{50}$ was calculated for fractions showing $>50 \%$ mortality using Probit analysis

\begin{tabular}{|c|c|c|c|c|c|}
\hline Compounds & $\begin{array}{l}\mathrm{LC}_{50} \\
(\mathrm{mg} \\
\left.\mathrm{L}^{-1}\right)\end{array}$ & $\begin{array}{l}\text { Confidence limits (mg } \\
\mathrm{L}^{-1} \text { ) }\end{array}$ & $\begin{array}{l}\text { Slope } \pm \\
\text { SE }\end{array}$ & $\begin{array}{l}\text { Chi } \\
\text { square }\end{array}$ & $\begin{array}{l}P \\
\text { value }\end{array}$ \\
\hline $\begin{array}{l}\text { Kaempferol-3-0-glucoside } \\
(72 \mathrm{~h})\end{array}$ & 4512.54 & $3455.14-6789.22$ & $\begin{array}{l}2.02 \pm \\
0.31\end{array}$ & 0.94 & 0.82 \\
\hline $\begin{array}{l}\text { Kaempferol-3-0-glucoside } \\
(96 \mathrm{~h})\end{array}$ & 3762.69 & $2924.95-5398.97$ & $\begin{array}{l}1.95 \pm \\
0.28\end{array}$ & 1.36 & 0.72 \\
\hline $\begin{array}{l}\text { Quercetin-3-Oglucoside } \\
\text { (72h) }\end{array}$ & 3068.62 & $2462.99-4093.71$ & $\begin{array}{l}2.09 \pm \\
0.28\end{array}$ & 2.21 & 0.53 \\
\hline $\begin{array}{l}\text { Quercetin-3-Oglucoside } \\
\text { (96h) }\end{array}$ & 1855.93 & $1550.48-2261.15$ & $\begin{array}{l}2.39 \pm \\
0.27\end{array}$ & 2.60 & 0.46 \\
\hline Gallic acid (72h) & 2339.69 & $1928.91-2933.01$ & $\begin{array}{l}2.24 \pm \\
0.27\end{array}$ & 2.03 & 0.57 \\
\hline Gallic acid (96h) & 1303.68 & $1118.34-1520.76$ & $\begin{array}{l}3.07 \pm \\
0.32\end{array}$ & 1.44 & 0.70 \\
\hline Shikimic acid (72h) & 2826.31 & $2320.31-3606.86$ & $\begin{array}{l}2.30 \pm \\
0.29\end{array}$ & 1.68 & 0.64 \\
\hline Shikimic acid (96h) & 1725.09 & $1447.64-2080.49$ & $\begin{array}{l}2.47 \pm \\
0.27\end{array}$ & 0.23 & 0.97 \\
\hline Azadirachtin (72 h) & 2642.32 & $2013.70-3816.64$ & $\begin{array}{l}1.53 \pm \\
0.22\end{array}$ & 0.99 & 0.80 \\
\hline Azadirachtin (96 h) & 1174.22 & $973.61-1416.60$ & $\begin{array}{l}2.28 \pm \\
0.25\end{array}$ & 5.16 & 0.16 \\
\hline
\end{tabular}

Detoxification enzyme activities of LEE, BEE and SO against A. craccivora

Detoxifying enzymes (GST and AChE) activities in A. craccivora fed with bean leaf discs treated with different concentrations of LEE, BEE and SO is presented in Fig. 1. Results showed that AChE activity of nymphs of $A$. craccivora indicated that all the concentrations of LEE, BEE and SO significantly inhibit the AChE activity $\left(F_{4,14}=631.00\right.$ to $\left.968.50, p<0.0001\right)$ as compared to control. However, LEE, BEE and SO at $2 \%$ reported higher inhibition of AChE $(0.19 \pm 0.00$ to $0.75 \pm 0.30 \mathrm{mU} / \mathrm{mg})$ and was followed by $1 \%$ $(0.28 \pm 0.20$ to $1.45 \pm 0.13 \mathrm{mU} / \mathrm{mg})$. Among them, BEE $2 \%(0.19 \pm 0.00 \mathrm{mU} / \mathrm{mg})$ showed higher inhibition of AChE and was followed by SO and LEE $(0.42 \pm 0.03$ and $0.75 \pm 0.30 \mathrm{mU} / \mathrm{mg}$, respectively) as compared to lower concentrations (0.25-0.5\%) (Fig. 1a).

Similarly for GST assay, all the concentrations of LEE, BEE and SO significantly inhibit the GST activity $\left(F_{4,14}=31.79\right.$ to $\left.500.37, p<0.0001\right)$ as compared to control. Among them, BEE at $2 \%(5.37 \pm 0.50 \mathrm{mU} / \mathrm{mg})$ 
showed higher inhibition of GST and was followed by LEE and SO $(9.41 \pm 1.13$ and $35.01 \pm 0.63 \mathrm{mU} / \mathrm{mg}$, respectively) as compared to lower concentrations (0.25-0.5\%). However, LEE, BEE and SO at $2 \%$ reported higher inhibition of GST (5.37 \pm 0.50 to $35.01 \pm 0.63 \mathrm{mU} / \mathrm{mg})$ and was followed by $1 \%(7.77 \pm 0.11$ to $41.26 \pm 1.88 \mathrm{mU} / \mathrm{mg}$ ) (Fig. 1b).

\section{Discussion}

In the current study, residual toxicity of leaf/bark extracts, seed oil, fractions, isolated compounds and its combinations (extracts with seed oil); synergistic activity of leaf/ bark extract with seed oil against $A$. craccivora is discussed. All the extracts/fractions/compounds/seed oil of $T$. sebifera showed toxicity against nymphs of $A$. craccivora. In the case of leaf extracts, the LEE $80 \%$ was found most effective $\left(\mathrm{LC}_{50}=6756.42 \mathrm{mg} \mathrm{L}^{-1}\right)$ and was followed by LME 80\% as compared to other extracts after $96 \mathrm{~h}$ of treatment. Our results agree with the findings of Ahmed et al. ${ }^{17}$ who reported, the ethanolic leaf extract of Citrullus colocynthis against Brevicornye brassicae $\left(\mathrm{LC}_{50}=10400 \mathrm{mg} \mathrm{L}^{-1}\right)$ after $72 \mathrm{~h}$ but not superior to our studies. Similarly, leaf ethanolic extract of Ricinus communis was more promising $\left(\mathrm{LC}_{50}=553 \mathrm{mg} \mathrm{L}-1\right.$; $58.6 \%$ mortality) against Myzus persicae ${ }^{18}$.In other studies, leaf methanolic extracts of Prosopis juliflora at $1 \%$ also showed $86.6 \%$ mortality against $A$. craccivora ${ }^{19}$.Similarly, among the bark extracts, BEE $80 \%$ $\left(\mathrm{LC}_{50}=5115.98 \mathrm{mg} \mathrm{L}^{-1}\right)$ was found to be more effective and was followed by BEE 100\% as compared to others after $96 \mathrm{~h}$ of treatments. The present study also confirms the findings of the previous report, where bark methanol extract of Prosopis juliflora at 1\% showed 93.3\% mortality against $A$. craccivora $^{19}$.

The SO of T. sebifera in the current study was found to be more effective after $72 \mathrm{~h}$ and $96 \mathrm{~h}$ of treatment $\left(\mathrm{LC}_{50}=2504.59\right.$ and $850.94 \mathrm{mg} \mathrm{L}^{-1}$, respectively) than leaf and bark extracts. Our results are agreed with the findings of Fenigstein et al. ${ }^{20}$, who reported seed oil of Ricinus communis (1.5\%) showed $75 \%$ mortality of Bemisiatabaci as compared to the present study where seed oil of $T$. sebifera recorded higher mortality against aphids at a lower concentration. In a similar study, neem seed oil $2 \%$ also showed a $71 \%$ reduction in the population of Lipaphis erysimi ${ }^{21}$. The insecticidal activity of SO of T. sebifera in the current study may be due to the presence of unsaturated fatty acids (oleic, linoleic and palmitoleic acid).

The residual toxicity of thecombination of SO with $80 \%$ LEE/BEE of $T$. sebifera against $A$. craccivorashowed significant synergistic interaction underlaboratory conditions. Among the combinations, $\mathrm{BEE}+\mathrm{SO}$ (1:1 ratio) was found to be more effective $\left(\mathrm{LC}_{50}=144.26 \mathrm{mg} \mathrm{L}^{-1}\right)$ against $A$. craccivoraand was followed by SO+LEE at 1:1 ratio, LEE+SO+BEE at $\{(1+1): 2\}$ ratio, LEE $+B E E$ at 1:3 ratio as compared to other mixtures $96 \mathrm{~h}$ after treatment under laboratory conditions. Similarly, under plant growth chamber, the binary mixtures of SO+LEE $\left(\mathrm{LC}_{50}=223.82 \mathrm{mg} \mathrm{L}^{-1}\right)$ was were more effective against A. craccivora and was followed by SO+BEE as compared to LEE, SO, and BEE. Based on FEI values, SO+BEE showed synergistic interaction. 
Among the among leaf fractions, $n$-hexane fraction $\left(\mathrm{LC}_{50}=196.61 \mathrm{mg} \mathrm{L}^{-1}\right)$ was the most promising against $A$. craccivora as compared to other fractions. However, among the bark fractions, ethyl acetate fraction was most effective $\left(\mathrm{LC}_{50}=813.45 \mathrm{mg} \mathrm{L}^{-1}\right)$ against $A$. craccivora. The efficacy of $n$-hexane fraction of leaf against $A$. craccivora may be due to the presence of metabolites like $n$-hexadecanoic acid, galaxolide, ethyl phthalate, octadecanoic acid, ethyl ester, etc. Similarly, $n$-hexane fraction of bark, the predominant metabolites are galaxolide followed by ethyl phthalate and 1-octadecene. It is also confirmed that the efficacy of tested fractions against the $A$. craccivora also may be due to the presence of flavonoids (kaempferol-3-0-glucoside, quercetin-3-0-glucoside) and phenolic acids (gallic acid and shikimic acid). In the current study, $n$-hexane leaf fraction showed strong efficacy against $A$. craccivora as compared to $n$ hexane fraction of Ageratum houstonianumand Eupatorium adenophorum $\left(\mathrm{LC}_{50}=2881\right.$ $2590 \mathrm{mg} \mathrm{L}^{-1}$ ) after $96 \mathrm{~h}^{22-23}$. Present results also confirm the findings of other reports where leaf $n$ hexane fraction of Ricinus communis showed $92-96 \%$ mortality against Sipha flava and Melanaphis sacchari ${ }^{24-25}$.Similarly, ethyl acetate leaf fraction in the current study also reported promising $\left(\mathrm{LC}_{50}=367.75 \mathrm{mg} \mathrm{L}^{-1}\right)$ than ethyl acetate fraction of Trillium govanianum $\left(\mathrm{LC}_{50}=2186.3 \mathrm{mg} \mathrm{L}^{-1}\right)$ against A. craccivora after $96 \mathrm{~h}^{26}$.

The residual toxicity of the isolated compounds against $A$. craccivora showed gallic acid was most effective $\left(\mathrm{LC}_{50}=1303.68 \mathrm{mg} \mathrm{L}^{-1}\right)$ against $A$. craccivora and was followed by shikimic acid and quercetin3-0-glucoside as compared to kaempferol-3-0-glucoside. The present results on the efficacy of gallic acid confirmed with that of Punia et al. ${ }^{27}$ who reported the gallic acid at $3125 \mathrm{ppm}$ incorporated diet fed to the larvae of $S$. litura showed $70 \%$ larval mortality, adult emergence, and delayed developmental period. In the current study, quercetin and kaempferol also showed toxicity against aphid and these results also confirmed with findings of earlier studies where, kaempferol and quercetin from methanolic extract of Buddle jalbifloraHemsl., showed efficacy against $P$. xylostella $\left(\mathrm{LC}_{50}=1.454 \mathrm{mg} \mathrm{mL}^{-1}\right)$, Mythimna separata (74-77\% mortality) and antifeedant against $P$. xylostella $\left(\mathrm{AFC}_{50}=1.019 \mathrm{mg} \mathrm{mL}^{-1}\right)^{28}$.

Detoxification enzymes, such as GST, cytochrome P450 monooxygenases, and carboxyl/cholinesterase's, are well known for helping plant-feeding insects to maintain physiological roles by detoxifying xenobiotic substances ${ }^{29-30}$.Pesticides and poisonous secondary metabolites from host plants are examples of xenobiotic substances ${ }^{30}$.The plant extracts/fractions/essential oils have a variety of modes of action against insects, including neurotoxicity, insect growth/digestive enzyme inhibition, and inhibition GST and cytochrome P450 monooxygenase ${ }^{31}$. The AChE activities in A. craccivora fed with bean leaf discs treated with different concentrations of LEE, BEE and SO showed significant inhibition as compared to control aphid. Present results confirmed with the findings of Dolma et al. ${ }^{32}$ who reported Tagetes minuta oil showed inhibitory effect on AChE in P. xylostella. In a similar study, essential oils and synthesized nano-emulsion from Basilicumocimum L., Cuminum cyminum L., Origanum marjorana L. and Matricaria chamomilla L. showed decreased activity of AChE in A. craccivora ${ }^{33}$. Similarly for the GST assay, all the concentrations of LEE, BEE and SO significantly inhibit the GST activity as compared to control. Our results conform with the findings of Phankaenet al., ${ }^{34}$ who reported caffeine from Coffea arabica extract 
inhibits GST and carboxylesterase (CarE) in Triboliumcastaneum. Similarly, our previous study also showed that Tagetes minuta oil inhibits GST in P. xylostella ${ }^{32}$.

The current study concludes that extract from leaf and bark,fractions and isolated compoundsof $T$. sebiferacan beused for the control of $A$. craccivora. The insecticidal activity might be due to the presence of individual or combination action of extracts and seed oil of $T$. sebifera. Based on the literature survey, the efficacy of $T$. sebifera extracts/fractions/ isolated compounds and seed oil against A. craccivora as well as the chemical composition of seed oil and $n$-hexane fractions had not previously been reported earlier. Thus, this study is unique and executed for the first time to examine the leaf, bark extracts and seed oil of $T$. sebifera against $A$. craccivora.

\section{Conclusions}

In the present study,among leaf/bark extracts, BEE $80 \%$ and $100 \%$ were found to be most effective followed by BME $50 \%$ as compared to LEE and LME $80 \%$. The seed oil was found more effective than leaf and bark extracts. Among combinations, $\mathrm{BEE}+\mathrm{SO}$ (1:1 ratio) was most effective against $A$. craccivora followed by SO+LEE at 1:1 ratio, $L E E+S O+B E E$ at $(1+1): 2$ ratios and $L E E+B E E$ at 1:3 ratio. All the combinations/blends showed synergistic activity to $A$. craccivora but LEE $+B E E$ at 1:3, 3:1, and 1:1 ratio showed the most significant synergistic interaction. Among leaf/bark fractions, the $n$-hexane leaf fraction was most effective against aphids. Among pure compounds, gallic acid was found most effective. LEE, BEE and SO significantly inhibit the AChE and GST activity in A. craccivora. Based on our findings, the leaf extracts/seed oil can be recommended for the control of aphids in crop plants under greenhouse/field conditions.

\section{Declarations}

\section{Acknowledgments}

Authors thank Director, CSIR-IHBT, Palampur for the necessary infrastructure. IHBT communication number for this article is 4903. Shudh Kirti Dolma is grateful to CSIR-HRDG for Senior Research Fellow (Award No: 31/54(0148)/2019-EMR-I).The work has been filed for patent entitled "A herbal formulation useful as an insecticide and a process for the preparation thereof" (Application No. 202011048468 dated 4.11.2020, India).

\section{Declaration of Competing Interest}

All authors of this manuscript declare that have no conflict of interest.

\section{Author contributions}

S.K.D., Investigation, data curation, formal analysis, validation, roles/writing - original draft, P.P.S., formal analysis, S.G.E.R., Conceptualization, supervision, funding acquisition, formal analysis, writing -editing. 
All the authors reviewed the manuscript.

\section{Funding}

The authors are grateful to the Council of Scientific and Industrial Research (CSIR), New Delhi for funding the mission project "CSIR-Aroma Mission (HCP-0007)".

\section{References}

1. Laamari, M. \& Khelfa, L. Coeur d'Acier, A. Resistance source to cowpea aphid (Aphis craccivora Koch) in broad bean (Vicia faba L.) Algerian landrace collection. African journal of. biotechnology, 7, 24862490 (2008).

2. Fouad, E. A., Yousef, H. M. A., Abdallah, I. S. \& \&Kandil, M. A. Resistance monitoring and enzyme activity in three field populations of cowpea aphid (Aphis craccivora) from Egypt. Crop protection, 81, 163-167 (2016).

3. Jaryan, V. et al. Distribution characteristics of Sapium sebiferum (L.) Roxb. - an invasive tree species in Himachal Pradesh, Western Himalaya. Proc. Indian national science academy 79, 215-234(2013)

4. Breitenbeck, G. A. Chinese tallow trees as a biodiesel feedstock.Louisiana agriculture26-27(2009)

5. Kim, Y., Welt, B. A. \& Talcott, S. T. The impact of packaging materials on the antioxidant phytochemical stability of aqueous infusions of green tea (Camellia sinensis) and yaupon holly (Ilex vomitoria) during cold storage. Journal of agricultural and food chemistry, 59, 4676-4683 (2011).

6. Khan, M., Kumar, S. \& Hamal, I. A. Medicinal plants of sewa river catchment area in the northwest Himalaya and its implication for conservation. Ethnobotanical leaflets, 13, 1113-1137 (2009).

7. Duke, J. A. \& \&Ayensu, E. S. Medicinal Plants of Chin. 2 Vols 05 S., 1300 Strichzeichnungen. Reference Publ., Inc. Algonac. Michigan, 1985. ISBN 0-917266-20-4. Preis: geb. m. Schutzumschlag $\$ 94,95(1985)$

8. Gao, Y., Ho, G., He, X. \& Chen, C. Studies on in vitro antioxidant activities of leaf extracts of Sapium sebiferum. Food science, 24, 141-145 (2003).

9. Huang, B. et al. Analgesic and anti-inflammatory effect of extract of Sapium sebiferum leaves on animal model. Chinese Traditional Patent Medicine, 26, 476-479 (2004).

10. Chaudhary, H. J. et al. Antimicrobial activities of Sapium sebiferum L. belonging to family Euphorbiaceae. Journal of medicinal plant research, 5, 5916-5919 (2011).

11. Fu, R. et al. Antioxidant and anti-inflammatory activities of the phenolic extracts of Sapium sebiferum (L.) Roxb. leaves. Journal of ethnopharmacology, 147, 517-524 (2013).

12. Taiwo, F. O., Oyedeji, O. \& \&Osundahunsi, M. T. Antimicrobial and antioxidant properties of kaempferol-3-0-glucoside and 1-(4-Hydroxyphenyl)-3-phenylpropan-1-one isolated from the leaves of Annona muricata (Linn.). Journal of pharmaceutical research international, 26, 1-13 (2019).

13. Lu, Y. \& Foo, L. Y. Identification and quantification of major polyphenols in apple pomace. Food chemistry, 59, 87-194 (1997). 
14. Pan, H. A non-covalent dimer formed in electrospray ionization mass spectrometry behaving as a precursor for fragmentations. Rapid Commun. Mass spectrometry, 22, 3555-3560 (2008).

15. Chanwitheesuk, A., Teerawutgulrag, A. \& Kilburn, J. D. \&Rakariyatham, N. Antimicrobial gallic acid from Caesalpinia mimosoidesLamk. Food chemistry, 100, 1044-1048 (2007).

16. Edmonds, M. \& Payne, R. Isolation of shikimic acid from star aniseed. Journal of chemical education, 82, 599-600 (2005).

17. Ahmed, M. et al. Insecticidal activity and biochemical composition of Citrullus colocynthis, Cannabis indica and Artemisia argyi extracts against cabbage aphid (Brevicoryne brassicae L.). Scientific reports, 10, 522-532 (2020).

18. Madanat, H. M., Antary, T. M. A. \& Zarqa, M. H. A. Toxicity of six ethanol plant extracts against the green peach aphid Myzuspersicae SULZER (Homoptera: Aphididae). Fresenius environmental bulletin, 25, 706-718 (2016).

19. Zerihun, M. \& \&Ele, E. Bioactive compounds and biological assays of different Prosopis juliflora extracts against groundnut aphid. Open chemistry, 6, 021-029 (2020).

20. Fenigstein, A., Eliyahu, M. \& Gan-Mor, S. Veierov, D. Effects of five vegetable oils on the sweetpotato whitefly Bemisiatabaci. Phytoparasitica, 29, 197-206 (2001).

21. Dotasara, S. K., Agrawal, N., Singh, N. \& Swami, D. Efficacy of some newer insecticides against mustard aphid LipaphiserysimiKalt. in cauliflower. Journal of entomology and zoology studies, $\mathbf{5}$, 654-656 (2017).

22. Adebisi, O., Dolma, S. K., Verma, P. K., Singh, B. \& Reddy, S. G. E. Volatile, non-volatile composition and insecticidal activity of Eupatorium adenophorumSpreng against diamondback moth, Plutella xylostella (L.), and aphid, Aphis craccivora Koch. Toxin reviews38, 143-150 (2019a)

23. Adebisi, O., Dolma, S. K., Verma, P. K., Singh, B. \& Reddy, S. G. E. Volatile, non volatile composition and biological activities of Ageratum houstonianum mill. against diamondback moth, Plutella xylostella (L.) and aphid, Aphis craccivora Koch. Indian journal of experimental biology, 57, 908-915 (2019b).

24. Sanchez, D. O. S. et al. Bioactivity of a fraction rich in linoleic acid of Ricinus communis L. (Euphorbiaceae) leaves against the yellow sugarcane aphid, Sipha flava (Hemiptera: Aphididae).Journal of food protection(2021) https://doi.org/10.4315/JFP-21-055.

25. Semnani, K. M., Akbarzadeh, M. \& \&Changizi, S. Essential oils composition of Stachys byzantina, $S$. inflata, S. lavandulifolia and S. laxa from Iran. Flavour and fragrance journal 21, 300-303 (2006)

26. Sotelo-Leyva, C. et al. Insecticidal compounds in Ricinus communis L. (Euphorbiaceae) to control Melanaphissaccharizehntner (Hemiptera: Aphididae). Florida entomologist 103,91-95(2020)

27. Dolma, S. K., Suresh, P. S., Singh, P. P., Sharma, U. \& Reddy, S. G. E. Insecticidal activity of the extract, fractions, and pure steroidal saponins of Trillium govanianum Wall. ex D. Don for the control of diamondback moth (Plutella xylostella L.) and aphid (Aphis craccivora Koch). Pest management science, 77, 956-962 (2020). 
28. Punia, A. et al. Effect of gallic acid on the larvae of Spodoptera litura and its parasitoids Braconhebetor.Scientific reports(2021) https://doi.org/10.1038/s41598-020-80232-1.

29. Zhang, X. Y., Shen, J., Zhou, Y., Wei, Z. P. \& Gao, J. M. Insecticidal constituents from BuddlejaalbifloraHemsl. Natural product research, 31, 1446-1449 (2017).

30. Feyereisen, R. 8 - Insect CYP Genes and P450 Enzymes. Editor: Lawrence, I. G. Insect molecular biology and biochemistry,Academic press,236-316(2012)

31. Oakeshott, J., Claudianos, C., Campbell, P. M., Newcomb, R. D. \& Russell, R. J. Biochemical genetics and genomics of insect esterases. Comprehensive reviews in food science and food safety, 5, 309381 (2005).

32. Park, Y. L. \& \&Tak, J. H. Essential oils for arthropod pest management in agricultural production systems. In book: Essential oils in food preservation, flavor and safety. (2016) DOI: 10.1016/B978-012-416641-7.00006-7

33. Dolma, S. K., Jayaram, C. S., Chauhan, N. \& Reddy, S. G. E. (2021) Effect of Tagetes minuta oil on larval morphology of Plutella xylostella through scanning electron microscopy and mechanism of action by enzyme assay. Toxin reviews (2021) DOI:10.1080/15569543.2021. 1988980

34. Phankaen, Y. et al. Toxicity and repellent action of Coffea arabica against Triboliumcastaneum (Herbst) adults under laboratory conditions. Journal of stored products research, 71, 112-118 (2017).

35. Abdelaal, K. et al. Toxicity of essential oils nanoemulsion against Aphis craccivora and their inhibitory activity on insect enzymes.Processes(2021). https://doi.org/10.3390/pr9040624

36. Stein, S. E. Mass spectral database and software, version 3.02, National Institute of Standards and Technology (NIST), Gaithersburg, MD, USA (1990)

37. Adams, R. P. Identification of essential oil components by gas chromatography/mass spectroscopy (Allured publishing corporation carol stream, Illinois, IL, 2007).

38. Rattan, R. et al. Triterpenoid saponins from Clematis graveolens Lindl. and evaluation of their insecticidal activity. Natural product communications, 10, 1525-1528 (2015).

39. Houghton, P. Synergy and polyvalence: paradigms to explain the activity of herbal products. In: Mukherjee, P.K. and Houghton, P. (Eds). Evaluation of herbal medicinal products: Perspectives on quality, safety and efficacy, London:Pharmaceutical Press,London, 85-94(2009)

40. Bassole, I. H. N. et al. Composition and antimicrobial activities of Lippia multiflora Moldenke, Mentha $x$ piperita L. and Ocimumbasilicum L. essential oils and their major monoterpene alcohols alone and in combination. Molecules 15, 7825-7839(2010)

41. Finney, D. J. Probit Analysis 3rd edn (Cambridge University Press, Cambridge, UK, 1971).

42. Jain, N., Srivastava, S. K., Aggarwal, K. K., Ramesh, S. \& Kumar, S. Essential oil composition of Zanthoxylum alatum seeds from northern India. Flavour and fragrance journal, 16, 408-410 (2001).

43. Semnani, K. M., Saeedi, M. \& \&Akbarzadeh, M. Essential oil composition of Teucrium scordium L. Acta pharmaceutica, 57, 499-504 (2007). 
44. Senatore, F., Arnold, N. A. \& Bruno, M. Volatile components of Centaurea eryngioides Lam. and Centaurea liberica Trev. var. hermonisBoiss. Lam., two Asteraceae growing wild in Lebanon. Natural product research, 19, 749-754 (2005).

45. Pinkston, D., Spiteller, G., Henning, H. \& Matthaei, D. High-resolution gas chromatography-mass spectrometry of the methyl esters of organic acids from uremic hemofiltrates. Journal of chromatography, 223, 1-19 (1981).

46. Rout, P. K., Misra, R., Sahoo, S., Sree, A. \& Rao, Y. R. Extraction of kewda (Pandanus fascicularisLam.) flowers with hexane: composition of concrete, absolute and wax. Flavour and fragrance journal, 20, 442-444 (2005).

47. Mosayebi, M. et al. Effect of habitat on essential oil of Achillea filipendula L. in Iran. Asian journal of plant sciences, 7, 779-781 (2008).

48. Viña, A. \& Murillo, E. Essential oil composition from twelve varieties of basil (Ocimum spp.) grown in Columbia. Journal of the brazilian chemical society, 14, 744-749 (2003).

49. Seo, W. H. \& \&Baek, H. H. Identification of characteristic aroma-active compounds from water dropword (Oenanthe javanica DC.). Journal of agricultural and food chemistry, 53, 6766-6770 (2005).

50. Andrade, E. H. A., Santos, A. S., Zoghbi, M. G. B. \& Maia, J. G. S. Volatile constituents of fruits of Astrocarium vulgare Mart. and Bactris gasipaes H.B.K. (Arecaceae). Flavour and fragrance journal 13, 151-153 (1998)

51. Woerdenbag, H. J., Windono, T., Bos, R., Riswan, S. \& \&Quax, W. J. Composition of the essential oils of Kaempferia rotunda L. and Kaempferia angustifolia Roscoe rhizomes from Indonesia. Flavour and fragrance journal, 19, 145-148 (2004).

52. Shen, X., Gao, Y. \& \&Su, Q. D. Constituents of the essential oil of Rhizoma polygonate. Flavour and fragrance journal, 21, 556-558 (2006).

53. Senatore, F., Landolfi, S., Celik, S. \& Bruno, M. Volatile components of Centaurea calcitrapa L. and Centaurea sphaerocephala L. ssp. sphaerocephala, two Asteraceae growing wild in Sicily. Flavour and fragrance journal, 21, 282-285 (2006).

54. Gómez, E., Ledbetter, C. A. \& Hartsell, P. L. Volatile compounds in apricot, plum, and their interspecific hybrids. Journal of agricultural and food chemistry, 41, 1669-1676 (1993).

55. Shiota, H. New esteric components in the volatiles of banana fruit (Musa sapientum L.). Journal of agricultural and food chemistry, 41, 2056-2062 (1993).

56. Egolf, L. M. \& Jurs, P. C. Quantitative structure-retention and structure-odor intensity relationships for a diverse group of odor-active compounds. Analytical chemistry, 65, 3119-3121 (1993).

\section{Figures}




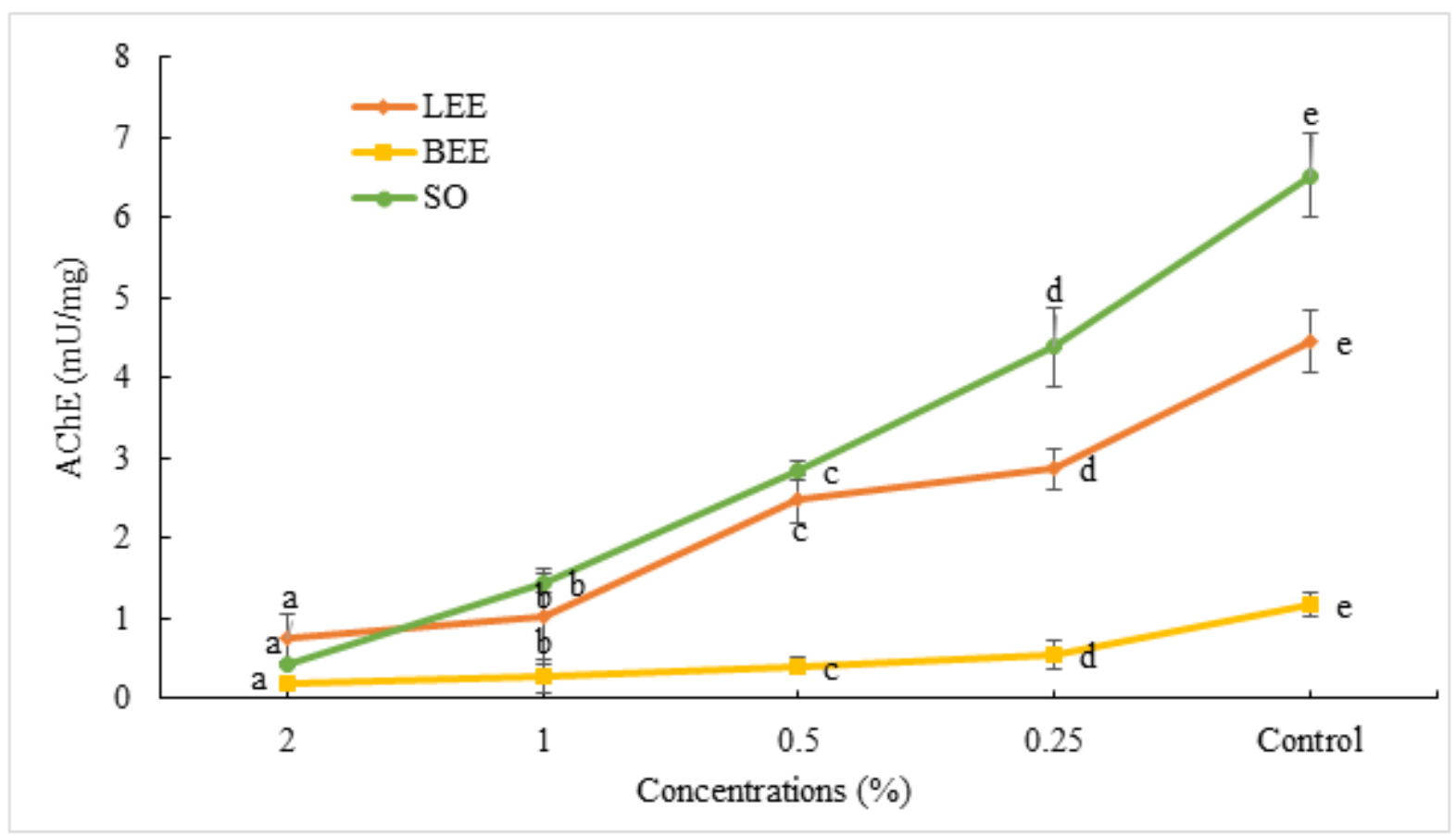

Fig.1a

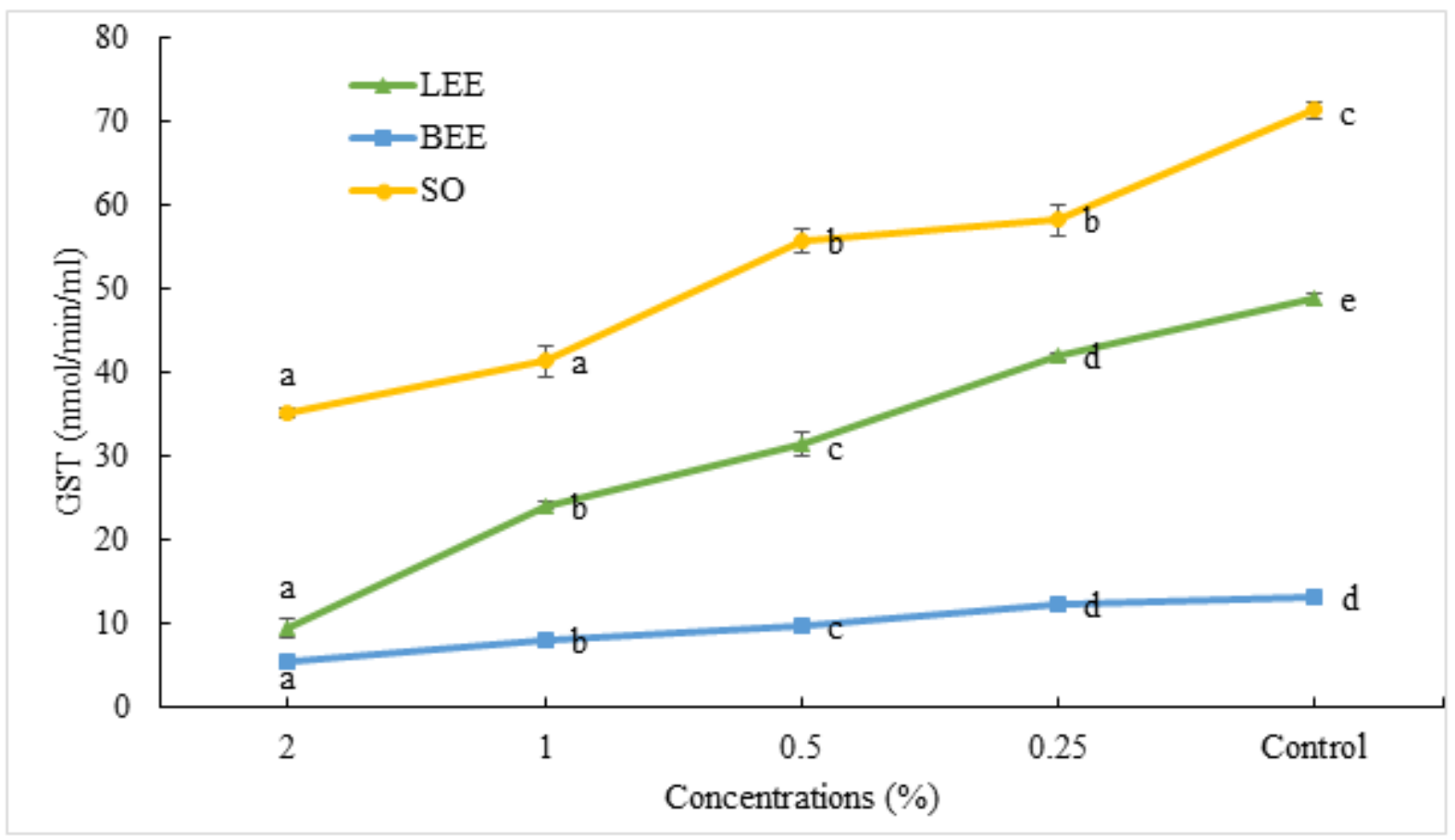

Fig. 1b

\section{Figure 1}

Detoxification enzyme activities: AChE (1a) and GST inhibition (1b) in Aphis craccivora treated with leaf extract, bark extract and seed oil of Triadica sebifera; Bars represents standard error ( \pm SE) of three replications. 


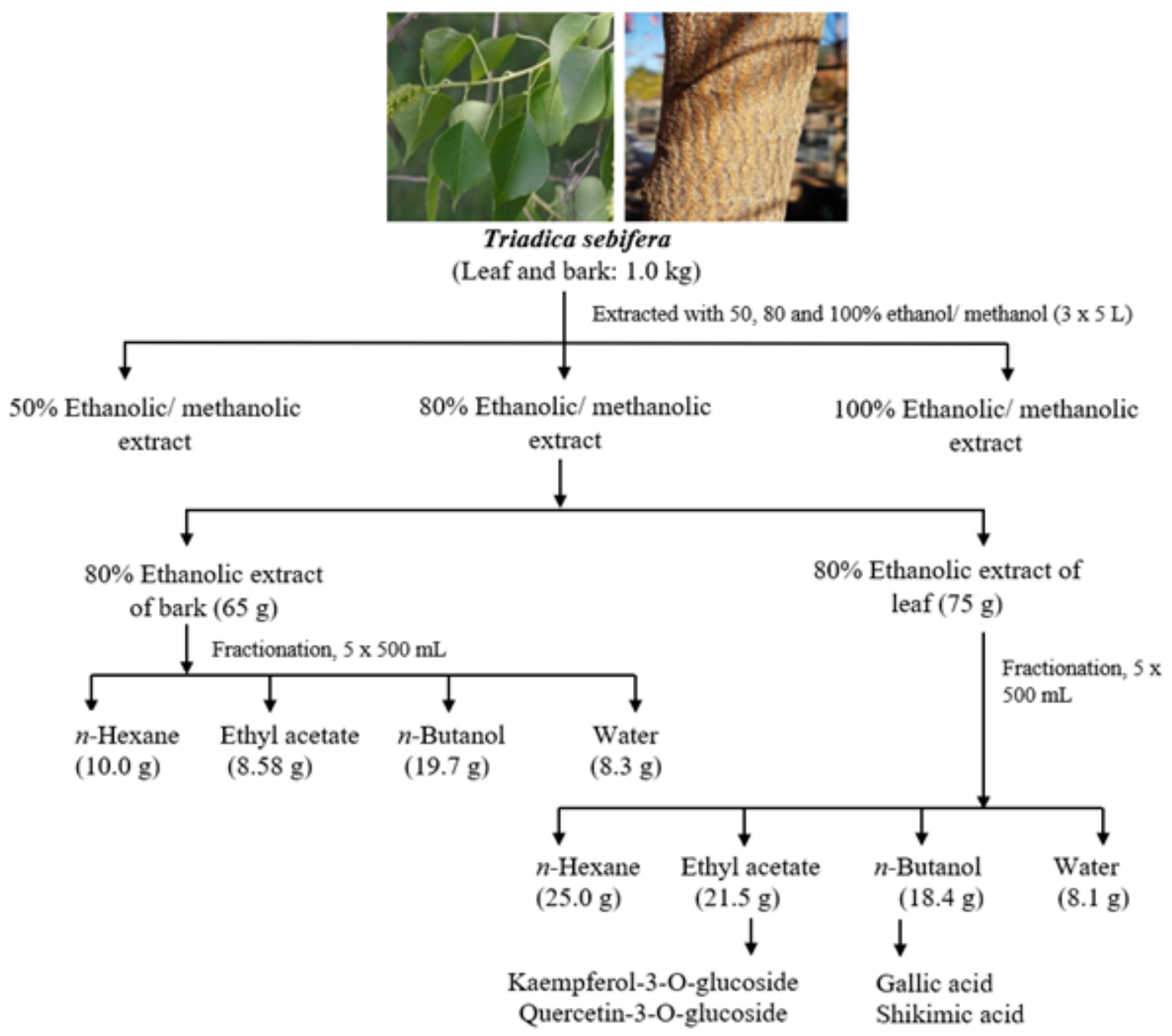

Figure 2

Schematic representation of extraction and fractionation of Triadica sebifera

\section{Supplementary Files}

This is a list of supplementary files associated with this preprint. Click to download.

- Supplementaryinformation.docx 